\title{
Thymoma and Tuberculoma: Unexpected Coexistence
}

\author{
António Grilo Novais ${ }^{1}$, Diana Pinho Santos ${ }^{1}$, Mariana Conceição ${ }^{2}$, Sara Cunha ${ }^{3}$, Joana Capelo ${ }^{1}$, Pedro Crespo $^{4}$, Lina Carvalho $^{5}$ \\ ${ }^{1}$ Internal Medicine Department, Centro Hospitalar Tondela-Viseu, Portugal \\ ${ }^{2}$ Pneumology Department, Centro Hospitalar Tondela-Viseu, Portugal \\ ${ }^{3}$ Thoracic Surgery Department, Centro Hospitalar e Universitário de Coimbra, Portugal \\ ${ }^{4}$ Infectious Diseases Department, Centro Hospitalar Tondela-Viseu, Portugal \\ ${ }^{5}$ Institute of Anatomical and Molecular Pathology, Faculty of Medicine, Coimbra University Hospital Center, Coimbra, Portugal
}

\section{Doi: 10.12890/2021_002744 - European Journal of Case Reports in Internal Medicine - @ EFIM 2021}

Received: 24/06/2021

Accepted: $12 / 07 / 2021$

Published: $30 / 07 / 2021$

\begin{abstract}
How to cite this article: Grilo Novais A, Pinho Santos D, Conceição M, Cunha S, Capelo J, Crespo P, Carvalho L. Thymoma and tubercoloma: unexpected
\end{abstract} coexistence. EJCRIM 2021;8: doi:10.12890/2021_002744.

Conflicts of Interests: The authors declare there are no competing interests.

This article is licensed under a Commons Attribution Non-Commercial 4.0 License

\section{ABSTRACT}

Mediastinal tumours can be incidental findings on chest x-ray or present with systemic symptoms and/or direct effect of the mediastinal mass. We report the case of a woman with symptomatic thymoma B1 and simultaneous thymus tuberculosis.

\section{LEARNING POINTS}

- $\quad$ The association of tuberculosis (TB) of the thymus and thymoma is extremely rare.

- The differential diagnosis of a mediastinal mass should include TB, particularly in endemic regions.

- Mediastinal mass resection or biopsy can be of great value in diagnostic work-up.

\section{KEYWORDS}

Thymus, tuberculosis, granuloma, mediastinal mass

\section{INTRODUCTION}

Thymomas are considered rare tumours ${ }^{[1]}$. In adults over 40 years of age, anterosuperior mediastinal masses are usually of thymic origin, with the majority being thymomas followed by germ cell tumours ${ }^{[2]}$. Tuberculosis of the thymus has rarely been reported as, to the authors' knowledge, only 15 cases had been previously reported worldwide ${ }^{[3]}$. The coexistence of these two diseases is even rarer ${ }^{[3]}$.

\section{CASE DESCRIPTION}

A 72-year-old Brazilian woman living in Portugal presented to the emergency department with non-exertional chest pain, worsening shortness of breath and asthenia for the previous 3 months.

She denied having cough, fever, weight loss or night sweats. She had not travelled abroad recently and had no recognized contacts with tuberculosis. Her history revealed that she had immigrated from Brazil 2 years previously and was uncertain as to her previous exposure to TB. She had received the BCG vaccine as a child.

Physical examination revealed no relevant abnormalities except for a muscle strength score of 3/5 in the upper limbs. Chest x-ray (Fig. 1) on admission showed superior mediastinal widening. Thorax computed tomography (CT) revealed an anterior mediastinal mass $(69 \times 62$ $\mathrm{mm}$ ), suggestive of thymoma. No additional vascular, lung or mediastinal changes were present (Fig. 2). The patient was admitted for further investigation. 


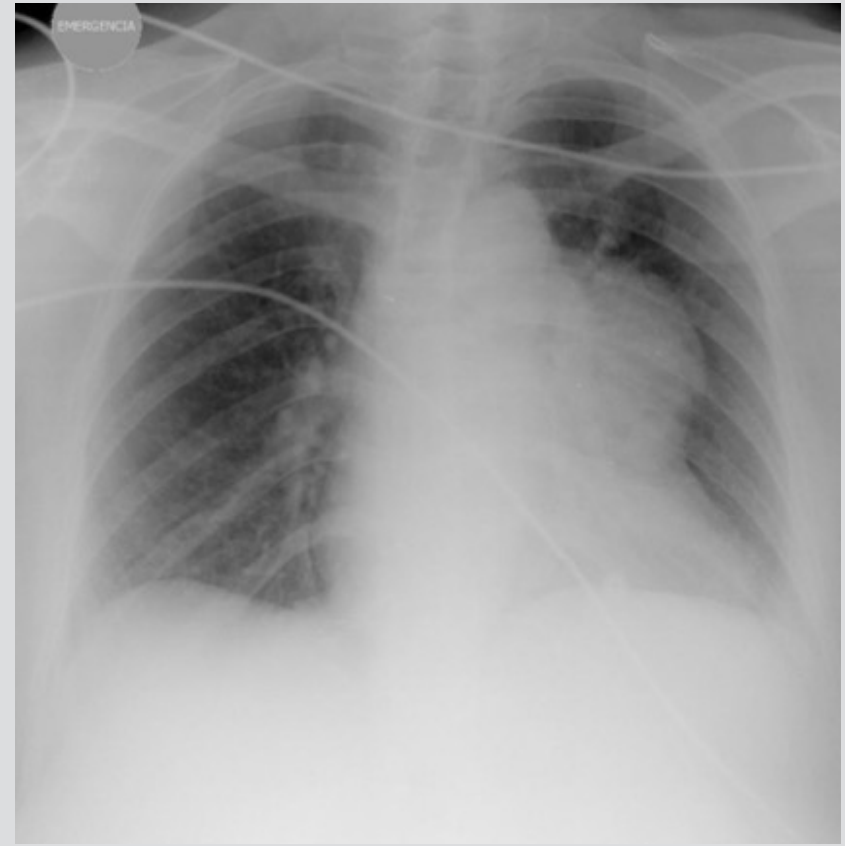

Figure 1. Mediastinal widening on chest $x$-ray

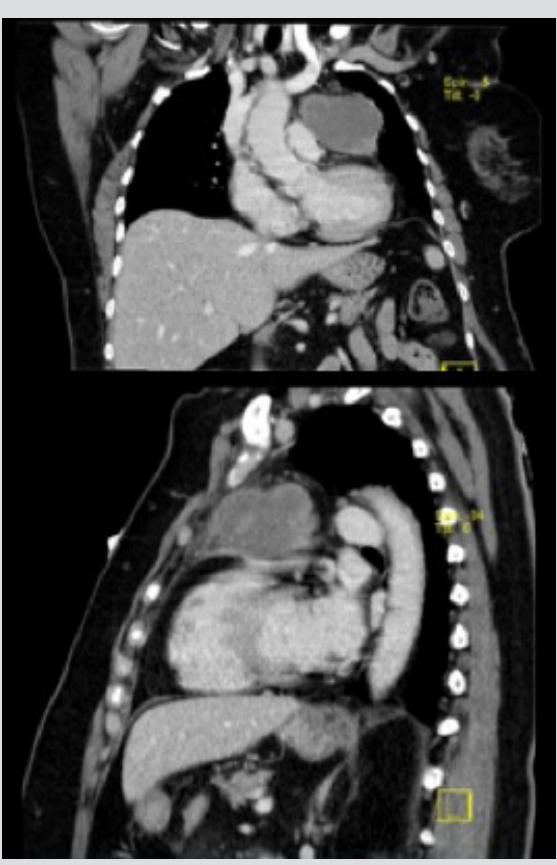

Figure 2. Computed tomography (CT) scan of the thorax showed an anterior mediastinal mass $(69 \times 62 \mathrm{~mm})$, suggestive of thymoma

Bedside tests, including ocular, palpebral and bulbar fatigability tests, were negative. Anti-acetylcholine receptor binding antibody, antiMuSK antibody and electromyography were negative, thus excluding myasthenia gravis. The patient was HIV negative.

As it was possible to resect the mass, the thoracic surgeon recommended thoracotomy. Complete surgical resection of the thymic mass was achieved. Macroscopically, the thymus presented a subcapsule nodular formation measuring $4 \times 4 \times 3.5 \mathrm{~cm}$, yellowish and soft where it was cut, as well as another $3.5 \mathrm{~cm}$ round nodular lesion, which was whitish and firm. Microscopically, the first yellowish lesion represented a tuberculoma with a central area of caseum surrounded by fibroblasts and scarce Langhans cells; acid fast bacilli were seen on Ziehl-Neelsen staining (Fig. 3C,D). The smaller lesion was classified as WHO type B1/B2 thymoma (Fig. 3A,B).

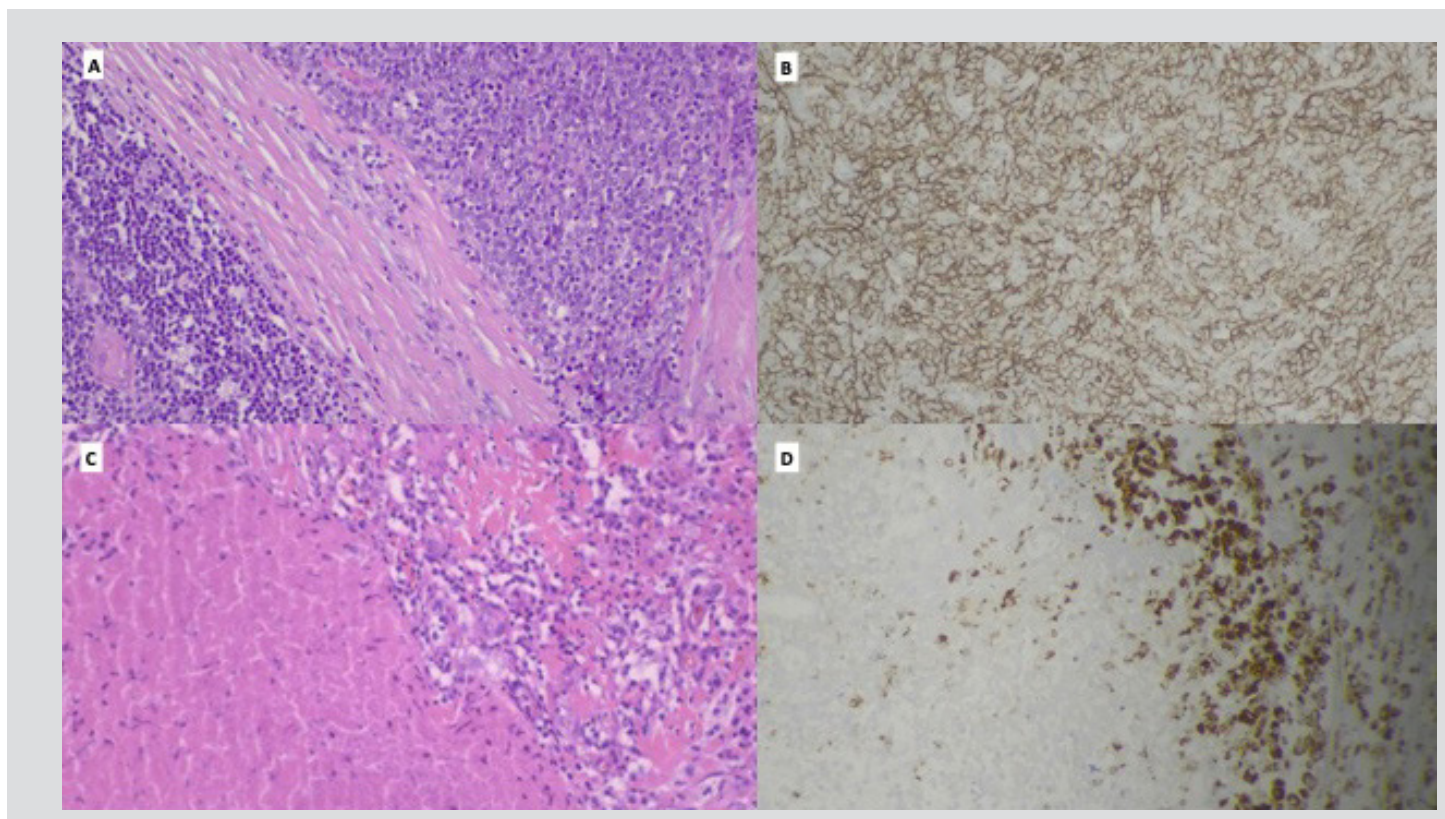

Figure 3. The $3.5 \mathrm{~cm}$ thymoma, B1/B2 subtyping, with stromal fibrotic bundles and typical moderate representation of small lymphocytes in a B2 pattern, also showing the cortical thymus with Hassall corpuscles $(A, H E \times 200)$ and evidence of large cuboidal epithelial cells (B, CK5.6×200). Tuberculoma with a maximum length of $4 \mathrm{~cm}$ with caseum and epithelioid macrophages and rare Langhans cells (C, $\mathrm{He} \times 200$ ) with CD68 expression (D, CD68 $\times 200$ ) temporal arteries (small arrows) 
The treatment of choice for any thymic tumour is based on the resectability of the tumour ${ }^{[4,5]}$. The present tumour was classified as MasaokaKoga stage I. The surgical excision was considered adequate and curative for the thymoma, and the patient was seen by the oncology department for follow-up. Her postoperative condition was satisfactory without serious complications.

The unexpected intrathymic tuberculosis which was also diagnosed required treatment with a standard 9-month antibiotic regimen (isoniazid, rifampicin, ethambutol and pyrazinamide). By the end of the treatment, the patient could tolerate mild exertion and had no further episodes of shortness of breath. At follow-up after 12 months, there was no history of recurrence of symptoms, and a revaluation CT scan showed no mediastinal widening.

\section{DISCUSSION}

Although tuberculosis involvement of mediastinal nodes is common, TB of the thymus is extremely rare. Only a few cases have been reported, with this being the 16th to the best of the authors' knowledge [6]. The association with thymoma is even rarer.

The symptoms in previously reported cases of thymic tuberculosis included non-specific general symptoms, including retrosternal chest pain, cough and progressive dyspnoea.

In Europe, Siesling et al. have found that the standardized incidence rate of thymic epithelial tumours is 0.17 per 100,000 population at risk. The incidence was highest in patients aged 65 years and older, was lowest in Northern and Eastern Europe and was highest in Central and Southern European countries ${ }^{[7]}$.

Type B1 thymoma may also be classified as lymphocyte-rich thymoma, lymphocytic thymoma, predominantly cortical thymoma or organoid thymoma. The most common cells in the tumour are T/CD3+ lymphocytes, which are more numerous than tumoural cells. The prognosis for people with type B1 thymoma is good, with $90 \%$ of patients living at least 20 years after diagnosis ${ }^{[8]}$.

Thymic tuberculosis possibly represents the remnants of post-primary localized mediastinal lymphadenitis ${ }^{[9]}$.

In conclusion, thymic tuberculosis and thymoma are two rare causes of mediastinal masses but were found here in the same patient. Symptoms in other reported cases of thymic tuberculosis included retrosternal chest pain, cough and progressive dyspnoea. In our patient, coincidental asymptomatic infection with TB may have occurred previously without classic symptoms of TB.

\section{REFERENCES}

Davenport E, Malthaner RA. The role of surgery in the management of thymoma: a systematic review. Ann Thorac Surg 2008;86(2):673-684. Kpodonu J, Cook JL, Massad MG, Snow NJ. Tuberculosis of the thymus: a case report and review of the literature. Curr Resp Med Rev 2005;1:123. Pillai KR, Prakash M, Pai MR. Clinicopathological characteristics of thymic tuberculosis - a rare entity. J Clin Diagn Res 2016;10(9):ED13-ED15. Kużdżał J, Asamura H, Detterbeck FC, Goldstraw P. Lerut T, Thomas T, et al. ESTS Textbook of Thoracic Surgery: Volume 2. 1st ed. Cracow: Medycyna Praktyczna; 2015 LoCicero III J, Feins RH, Colson YL, Rocco G. Shields' General Thoracic Surgery: Volume One and Volume Two. 8th ed. Philadelphia; Wolters Kluwer; 2018.

Ruangnapa K, Anuntaseree W, Suntornlohanakul S. Tuberculosis of the thymus in a 6-month-old infant with literature review. Pediatr Infect Dis J 2014;33(2):210-212.

Siesling S, van der Zwan JM, Izarzugaza I, Jaal J, Treasure T, Foschi R, et al; RARECARE Working Group. Rare thoracic cancers, including peritoneum mesothelioma. Eur J Cancer 2012;48(7):949-960.

8. Kalhor N, Moran CA. Thymoma and thymic carcinoma: a perspective on the NCCN clinical practice guidelines in oncology. Mediastinum 2018;2:49.

9. WHO. Global tuberculosis report 2020. Geneva: World Health Organization; 2020. 